\title{
Intimate Partner Cyberstalking Scale (IPCS): Evidências Psicométricas no Brasil
}

\author{
Intimate Partner Cyberstalking Scale (IPCS): Psychometric Evidence in Brazil
}

\author{
Paulo Gregório Nascimento da Silva ${ }^{1}$, Patrícia Nunes da Fonseca ${ }^{2}$, Emerson Diógenes de Medeiros $^{3}$, \\ Ricardo Neves Couto ${ }^{4}$ e Rayssa Soares Pereira ${ }^{5}$
}

\begin{abstract}
Resumo
O cyberstalking, refere-se a um padrão de comportamento repetido, intencional e indesejado direcionado às vítimas. Considerando o contexto dos relacionamentos íntimos e o papel de agressor, verifica-se um maior envolvimento desse grupo em condutas antissociais e delitivas, entretanto, tal conduta ainda é pouco explorada pela literatura. Portanto, uma medida adequada para rastrear a perpetração do cyberstalking ajudará na compreensão da temática em diferentes contextos. Em dois estudos (N=467), foram avaliados os parâmetros psicométricos da Intimate Partner Cyberstalking Scale (IPCS) por meio de diferentes técnicas (e.g., Análise Fatorial Confirmatória, Teoria de Resposta ao Item), além de propor uma versão curta da escala. Ambas as versões apresentaram uma estrutura unidimensional confiável e apresentaram correlações semelhantes com maquiavelismo, psicopatia e narcisismo. No geral, os resultados mostram que a IPCS e sua versão curta são medidas úteis para a avaliação da perpetração do cyberstalking e seus correlatos no Brasil.
\end{abstract}

Palavras-chave: cyberstalking, personalidade sombria, validade, precisão

\begin{abstract}
Cyberstalking refers to a pattern of repeated behavior, intentional and unwanted directed at victims. Considering the context of intimate relationships and the role of aggressor, there is a greater involvement of this group in antisocial and criminal conduct, however, such conduct is still little explored in the literature. Therefore, an adequate measure to track the perpetration of cyberstalking will help in understanding the theme in different contexts. In two studies $(\mathrm{N}=467)$, the psychometric parameters of the Intimate Partner Cyberstalking Scale (IPCS) were evaluated using different techniques (e.g., Confirmatory Factor Analysis, Item Response Theory), and a short version of the scale proposed. Both versions had a reliable onedimensional structure and had similar correlations with machiavellianism, psychopathy, and narcissism. Overall, the results show that the IPCS and its short version are useful measures for assessing the perpetration of cyberstalking and its correlates in Brazil.
\end{abstract}

Keywords: cyberstalking, dark personality, validity, accuracy

O presente artigo contou com apoio do CNPq por meio da bolsa de produtividade ao segundo e terceiro autores, além da CAPES, que concedeu bolsas de doutorado ao primeiro autor e de mestrado a quinta autora. Aproveitamos para demonstrar nossa gratidão a essas instituições. Toda correspondência deverá ser encaminhada à Paulo Gregório Nascimento da Silva, telefone: (83) 99847-2799. Email: silvapgn@gmail.com; Universidade Federal da Paraíba. Castelo Branco, João Pessoa/PB. CEP: 58059-900. Centro de Educação, Departamento de Psicopedagogia.

\footnotetext{
${ }^{1}$ Mestre em Psicologia e doutorando em Psicologia Social pela Universidade Federal da Paraíba - UFPB, João Pessoa-PB, Brasil. Tel.: +55(83)99967-6859. E-mail: silvapgn@gmail.com

${ }^{2}$ Doutora em Psicologia Social. Professora da Pós-Graduação em Psicologia Social pela Universidade Federal da Paraíba - UFPB, Brasil. Email: patynfonseca@hotmail.com

${ }^{3}$ Doutor em Psicologia Social. Professor Adjunto do curso de graduação em Psicologia e do Programa de Pós-Graduação em Psicologia (Mestrado) da Universidade Federal do Delta do Parnaíba - UFDPAR, Brasil. E-mail: emersondiogenes@gmail.com

${ }^{4}$ Doutor em Psicologia Social e pós-doutorando do programa de Pós-Graduação em Psicologia da Universidade Federal do Delta do Parnaíba - UFDPAR, Brasil. E-mail: r.nevescouto@gmail.com

${ }^{5}$ Psicopedagoga e Mestranda em Psicologia Social pela Universidade Federal da Paraíba - UFPB, Brasil. E-mail: rayssasp95@ gmail.com

Revista Iberoamericana de Diagnóstico y Evaluación - e Avaliação Psicológica. RIDEP $\cdot$ No59 $\cdot$ Vol.2 $\cdot 5-17 \cdot 2021$

ISSN: 1135-3848 print /2183-6051online
} 


\section{Introdução}

A perseguição (compreendida como stalking) sempre existiu na sociedade, todavia a sua construção histórica de criminalização mudou nos últimos anos (Spitzberg \& Cupach, 2003). A legislação pioneira de stalking foi criada na Califórnia, em 1990, denominada de Lei Antiperseguição. Posteriormente foi adotada pelos demais estados americanos, além de outros países, a exemplo, da Austrália e do Reino Unido (Sheridan, Blaauw, \& Davies, 2003).

O stalking é definido como um padrão de comportamento de intimidação intencional, repetitivo e intrusivo direcionado a uma pessoa específica, com a finalidade de incomodar, ameaçar e amedrontar a vítima (Fox, Nobles, \& Fisher, 2011). É praticado por um indivíduo que possui uma obsessão por uma pessoa, com a qual insiste em começar ou manter uma relação com propósitos específicos (Pires, Sani, \& Soeiro, 2018). Esta perseguição ocorre principalmente contra parceiros íntimos (antigos e/ou atuais), resultando em sofrimento físico, psíquico e financeiro para a vítima (Smoker \& March, 2017).

Com o advento da tecnologia emerge uma nova forma de perseguição perpetrada, denominado de cyberstalking (March, Litten, Sullivan, \& Ward, 2020), que é caracterizado por um padrão de comportamento repetido, intencional $\mathrm{e}$ indesejado direcionado às vítimas (Spitzberg \& Cupach, 2007). A sua incidência foi verificada inicialmente por Bocij e McFarlane, (2003), que observaram, dentre cada quatro pessoas perseguidas, um relato de vítima de cyberstalking. Posteriormente, Tokunaga e Aune (2015) observaram que cerca de 20 a 40 por cento dos usuários da internet são vítimas de cyberstalking. Considerando, especificamente, a perspectiva do agressor, o estudo realizado por Reyns, (2018), com 1.310 universitários estadunidenses, observou que 4,9\% já havia praticado comportamentos de perpetração.

$\mathrm{O}$ aumento dos índices da prática do cyberstalking pode estar relacionado ao fato de haver uma facilidade do anonimato na internet, o que colabora para uma maior proliferação da agressão (Smoker \& March, 2017). Isto poderá promover comportamentos antissociais e/ou delituosos já que os agressores agem sem medo de retaliações (Bocij \& McFarlane, 2003). Ademais, o cyberstalking pode ainda ter sido favorecido pelos envios de pedidos de amizades e de mensagens nas redes sociais ou, até mesmo, por atos mais graves, tais como o envio de e-mails abusivos, ameaçadores ou obscenos à vítima ou seus familiares (DeTardo-Bora, \& Bora, 2016).

As ações dos agressores ocorrem em forma de assédio, perseguição e agressão em contexto online/por meios digitais (Smith-Darden Poco, Kernsmith, Victor, \& Lathrop, 2017). Com a internet, a perseguição se tornou ainda mais fácil, quando comparada aos métodos tradicionais de perseguição, pois o agressor online (Smoker \& March, 2017), pode ter acesso a informações detalhadas da vida da vítima, sobretudo se essa publica em redes sociais fatos da sua vida diária (Eterovic-Soric, Choo, Ashman, \& Mubarak, 2017).

Diante do que já foi explanado, ressalta-se a importância de estudar esta temática, sobretudo, atualmente, a completa disseminação do uso das tecnologias digitais, e, com isso, o crescimento do número de cyberstalkers bem como de vítimas. Deste modo, destaca-se que, a presente pesquisa pretende estudar o papel do agressor (cyberstalkers) nos relacionamentos íntimos, em virtude de haver um maior envolvimento destas pessoas em condutas antissociais e delitivas (Goodboy \& Martin, 2015) e por ainda ser pouco explorado pela literatura (Smoker \& March, 2017). Ademais, entende-se que o ato da agressão pode ser potencializado no cyberstalking, uma vez que existe o desconhecimento dos limites espaciais ou temporais, causando na vítima uma percepção de vulnerabilidade devido ao aumento da capacidade do perpetrador de manipular, coagir, controlar e assediar a vítima (Tokunaga, 2016).

Além do mais sabe-se que comumente o comportamento de stalkear alguém de forma online estar associado a um impulso obsessivo por perseguir alguém, além do uso excessivo e problemático das redes sociais (Wegmann, Oberst, Stodt, \& Brand, 2017), isto faz com que o agressor gaste bastante tempo investigando as publicações que são compartilhadas em diferentes contas e perfis de suas vítimas (Kircaburun, Demetrovics, \& Tosuntaş, 2018), que também está relacionado a necessidade de controlar os relacionamentos (March et al., 2020). 
Para além disso, considerando de forma específica o papel do agressor, Ménard e Pincus (2012) argumentam que pessoas que passaram por eventos traumáticos apresentam uma maior probabilidade de exibir comportamentos de perseguição, pois aprenderam a se relacionar com os demais de maneira abusiva e intrusiva, podendo apresentar estilos de apego inseguro, sintomas de ansiedades e conflitos relacionais, devido a busca por aprovação.

Pesquisadores têm procurado diferenças individuais para explicar a conduta agressiva (do cyberstalking), por exemplo na variável sexo (Smoker \& March, 2017; Tani, Greenman, Schneider, \& Fregoso, 2003). Assim, algumas variáveis têm sido consideradas, a exemplo do sexo. Porém, apesar dos homens serem percebidos como mais consistentes no comportamento de perpetração do cyberstalking (Ahlgrim \& Terrance, 2018), ainda não existe um consenso no que tange o contexto dos relacionamentos íntimos. Nessa direção, a pesquisa, levada a cabo por Smoker e March (2017), tem mostrado que as mulheres são mais propensas, em comparação aos homens, a se engajarem na perseguição dos parceiros no ambiente virtual. Entretanto, outras pesquisas sugerem não haver diferenças entre homens e mulheres na participação, tanto como vítimas quanto como perpetradores do cyberstalking (Dreßing, Bailer, Anders, Wagner, \& Gallas, 2014; Strawhun, Adams, \& Huss, 2013).

Destarte, em razão das consequências prejudiciais ocasionadas por essas manifestações agressivas; outra característica individual que tem se demonstrado importante para explicar esta conduta é a personalidade sombria, que emerge da combinação de quatro traços, reconhecidos como Dark Tetrad, a saber: personalidade, considerando a tríade sombria (psicopatia, maquiavelismo e narcisismo, além do sadismo (Smoker \& March, 2017). Em revisão sistemática, Moor e Anderson (2019) observaram que apesar do impacto dos traços sombrios na explicação de comportamentos online, ainda é pequeno o número de pesquisas que exploraram tal relação, principalmente considerando relacionamentos íntimos (Kircaburun et al., 2018).

Entretanto, deve-se ter em conta que existem poucos dados empíricos referentes à prática do cyberstalking, principalmente quando se busca instrumentos para sua mensuração nos relacionamentos íntimos. Devido a isto, Smoker e March (2017) elaboraram a Intimate Partner Cyberstalking Scale (IPCS) visando medir comportamentos de perpetração do cyberstalking. Para tanto, foram elaborados 21 itens derivados de medidas existentes. Ressalta-se que, durante a construção dos itens da IPCS, optou-se por não utilizar termos que provocassem culpa ou vergonha no respondente ao concordar com o item. Entretanto, apesar de apresentar meritória consistência interna $(\alpha=.92)$, a medida não foi posta à prova em estudo piloto ou validação (semântica e construto), sendo reunidas apenas evidências de validade convergente.

Por exemplo, a pesquisa inicial de Smoker e March (2017), com 689 participantes verificou que o sexo foi um preditor significativo da perpetração do cyberstalking em parceiros íntimos, com mulheres sendo mais propensas a se envolverem nesse comportamento. Outrossim, todos os traços da Dark Tetrad (maquiavelismo, narcisismo, psicopatia e o sadismo) foram preditores significativos do cyberstalking, possivelmente, isto possa ser explicado em função do anonimato e do desapego que são proporcionados pela internet, contribuindo para otimizar a natureza aversiva das características da personalidade sombria (Lyons, 2019). Posteriormente, estudo levado a cabo por March et al. (2020) corroborou o poder preditivo dos fatores da dark tetrad em comportamentos de cyberstalking em parceiro íntimo, além de evidenciar que perpetradores apresentam uma necessidade de controle do relacionamento.

Como anteriormente comentado, apesar de resultados preliminares sugerirem que $\mathrm{o}$ cyberstalking possa estar associado a outros comportamentos que comumente estão relacionados ao uso problemático das mídias sociais, a exemplo do cyberbullying e cybertrolling (Goodboy \& Martin, 2015; Kircaburun et al., 2018), pesquisas focadas no papel do agressor são escassas na literatura (Smoker \& March, 2017).

Posto isto, entende-se que investigações empíricas possibilitarão uma melhor compreensão deste comportamento e de estimativas quanto a sua prevalência e possíveis antecedentes. Para 
tanto, é necessário contar com instrumentos psicométricos adequados. Considerando isto, March et al. (2020) argumentam que, embora estudos prévios tenham atestado índices de precisão da IPCS, a sua estrutura fatorial permanece inexplorada, sendo necessária colocála a prova para a dimensionalidade do instrumento (Smoker \& March, 2017). Aliado a isto, sabe-se que averiguar as propriedades psicométricas da Intimate Partner Cyberstalking Scale (IPCS) no contexto brasileiro, pode suscitar pesquisas sobre a temática em âmbito nacional. Sendo assim, o presente estudo, visa adaptar o instrumento supracitado para o Brasil, reunindo evidências de validade (estrutura interna e convergente), precisão e parâmetros individuais dos itens (dificuldade, discriminação e informação psicométrica) da IPCS.

\section{Método}

Estudo 1. Adaptação e evidências de estrutura
interna da Intimate Partner Cyberstalking Scale.

\section{Participantes}

Participaram 242 pessoas residentes nos estados do Piauí (94.60\%), Paraíba (2.10\%), Ceará $(2.90 \%)$ e Maranhão (.40\%). Estes foram recrutados de maneira acidental, não probabilística $\left(M_{\text {idade }}=21.51 ; D P=3.71\right.$; amplitude 18 a 40 anos), atentando-se ao critério de inclusão: ter passado ou está em um relacionamento romântico com duração de pelo menos um mês. Assim, foi verificado que os participantes em sua maioria estavam namorando (46.7\%) e/ou casado/união estável (18.2), eram do sexo feminino (57.4\%), heterossexuais $(80 \%)$ e com ensino superior incompleto $(72.2 \%)$.

\section{Instrumentos}

Os participantes responderam a um livreto contendo os seguintes instrumentos:

Intimate Partner Cyberstalking Scale (IPCS; Smoker \& March, 2017). Trata-se de uma medida composta por 21 itens, que avalia de maneira global o cyberstalker. Tendo como exemplo os itens 04 “Já verifiquei mensagens de meu(minha) parceiro(a) (ex. e-mail, Facebook, celular) sem ele(a) saber." e o item 08 "Já verifiquei o histórico do telefone/computador do (a) meu (minha) parceiro (a) para verificar o que ele(a) estava fazendo" , que são respondidos em uma escala tipo likert de cinco pontos, variando de 1 (Discordo totalmente) a 5 (Concordo totalmente).

Questionário sociodemográfico: objetivando caracterizar a amostra, os participantes responderam a um conjunto de perguntas: sexo, idade, status de relacionamento, estado civil e nível de escolaridade, renda familiar.

\section{Procedimento}

Inicialmente, para traduzir a IPCS para o português foi considerado o método de tradução reversa back-translation (Borsa, Damásio, \& Bandeira, 2012). Assim, a escala foi traduzida para o português brasileiro, passando por traduções às cegas por dois pesquisadores bilíngues independentes, que em seguida retraduziram o instrumento para o inglês (idioma original), para comprovar a equivalência dos itens das duas versões (português e inglês). Os tradutores tiveram atenção tanto no processo de ajuste semântico quanto idiomático, fazendo, quando preciso, adequações e correções nos itens. Posteriormente, a versão brasileira preliminar da IPCS passou por um estudo piloto, para validação semântica (Pasquali, 2016), para avaliar se os itens e o formato da medida estavam compreensíveis e prontos para aplicação, não sendo verificada necessidade de modificações.

A coleta se deu em formato eletrônico, sendo disponibilizado aos participantes um link em redes sociais e aplicativos de mensagens (e.g., Facebook, Instagram, WhatsApp e Telegram). Nesse caso, foi utilizada a técnica de bola de neve para participação na pesquisa, que é considerado um método útil para acessando uma amostra de forma mais ampla (Ruiz-García et al., 2019). Aos que aceitaram colaborar, eram esclarecidos os propósitos e assegurados o anonimato e sigilo. A pesquisa seguiu as Resoluções $n^{\circ}$ 466/12 e 510/2016 do Conselho Nacional de Saúde, com projeto submetido e aprovado no Comitê de Ética em Pesquisa do Centro de Ciências da Saúde de uma instituição pública brasileira (Número do Parecer: 3.624.087/ CAAE: 20170719.6.0000. 5188). 
Quadrol. Estrutura Fatorial, discriminação, limiares e quantidade de informação da IPCS

\begin{tabular}{|c|c|c|c|c|c|c|c|c|c|c|c|}
\hline & \multirow{2}{*}{ Itens } & \multicolumn{3}{|c|}{ EFA Robusta } & \multicolumn{7}{|c|}{ Modelo de Resposta Graduada (TRI) } \\
\hline & & F1 & & & $\mathrm{h}^{2}$ & A & b1 & b2 & b3 & b4 & $\mathrm{I}(\theta)^{*}$ \\
\hline \multirow{21}{*}{$\frac{\mathscr{E}}{=}$} & I_09 & .722 & .607 & .801 & .885 & 2.091 & 2.091 & .813 & 1.749 & 2.365 & 2.700 \\
\hline & I_21 & .698 & .599 & .766 & .898 & 1.970 & 1.970 & .675 & 1.812 & 2.549 & 3.124 \\
\hline & I_20 & .695 & .580 & .776 & .711 & 1.927 & 1.927 & .146 & .957 & 1.293 & 2.460 \\
\hline & I_05 & .708 & .604 & .781 & .733 & 1.920 & 1.920 & .145 & 1.013 & 1.243 & 2.086 \\
\hline & I_08 & .719 & .618 & .794 & .805 & 1.846 & 1.846 & -.040 & .589 & .962 & 1.958 \\
\hline & I_19 & .689 & .581 & .770 & .979 & 1,799 & 1,799 & -.138 & 0,894 & 1,716 & 3.130 \\
\hline & I_06 & .684 & .593 & .760 & .786 & 1.610 & 1.610 & -1.412 & -.523 & .028 & 1.418 \\
\hline & I_14 & .642 & .513 & .730 & .688 & 1.587 & 1.587 & .012 & .935 & 1.543 & 2.442 \\
\hline & I_01 & .637 & .499 & .723 & .848 & 1.544 & 1.544 & -.916 & .181 & .872 & 2.094 \\
\hline & I_03 & .615 & .495 & .707 & .866 & 1.537 & 1.537 & -.123 & 1.000 & 1.868 & 2.576 \\
\hline & I_12 & .610 & .466 & .698 & .848 & 1.458 & 1.458 & .607 & 1.625 & 2.133 & 2.853 \\
\hline & I_13 & .604 & .486 & .708 & .785 & 1.399 & 1.399 & -.207 & .644 & 1.279 & 2.519 \\
\hline & I_04 & .643 & .531 & .727 & .970 & 1.380 & 1.380 & -.771 & .078 & .351 & 1.850 \\
\hline & I_17 & .595 & .473 & .685 & .812 & 1.321 & 1.321 & -.738 & .388 & 1.230 & 2.550 \\
\hline & I_02 & .591 & .469 & .685 & .827 & 1.267 & 1.267 & -.715 & .299 & .875 & 2.109 \\
\hline & I_16 & .568 & .416 & .665 & .910 & 1.208 & 1.208 & .559 & 2.056 & 4.135 & 4.477 \\
\hline & I_18 & .518 & .377 & .626 & .613 & 1.207 & 1.207 & .418 & 1.786 & 2.500 & 4.104 \\
\hline & I_07 & .532 & .392 & .632 & .748 & 1.098 & 1.098 & .180 & 1.033 & 1.368 & 2.373 \\
\hline & I_11 & .618 & .517 & .712 & .875 & 1.090 & 1.090 & -.338 & .867 & 1.605 & 3.384 \\
\hline & I_15 & .467 & .300 & .581 & .839 & 1.038 & 1.038 & 1.531 & 3.129 & 4.332 & 4.616 \\
\hline & I_10 & .593 & .461 & .694 & 1.000 & 1.024 & 1.024 & -.540 & .344 & .852 & 2.271 \\
\hline \multirow{10}{*}{$\begin{array}{l}\stackrel{\mathscr{J}}{=} \\
\stackrel{\theta}{\theta}\end{array}$} & I_09 & .742 & .637 & .824 & .550 & 2.182 & 2.182 & .802 & 1.720 & 2.324 & 2.660 \\
\hline & I_21 & .724 & .627 & .800 & .724 & 2.092 & 2.092 & .659 & 1.765 & 2.471 & 3.020 \\
\hline & I_20 & .728 & 609 & .806 & .728 & 2.026 & 2.026 & .138 & .953 & 1.288 & 2.419 \\
\hline & I_05 & .717 & .602 & .799 & .717 & 1.875 & 1.875 & .140 & 1.031 & 1.269 & 2.138 \\
\hline & I_08 & .683 & .547 & .771 & .466 & 1.698 & 1.698 & -.056 & .609 & 1.007 & 2.068 \\
\hline & I_19 & .671 & .544 & .766 & .451 & 1.631 & 1.631 & -.161 & .936 & 1.812 & 3.330 \\
\hline & I_06 & .686 & .568 & .767 & .471 & 1.604 & 1.604 & -1.419 & -.528 & .028 & 1.430 \\
\hline & I_14 & .625 & .486 & .719 & .391 & 1.476 & 1.476 & -.005 & .971 & 1.616 & 2.569 \\
\hline & I_01 & .608 & .470 & .699 & .370 & 1.388 & 1.388 & -.985 & .176 & .915 & 2.231 \\
\hline & I_03 & .624 & .511 & .726 & .390 & 1.509 & 1.509 & -.129 & 1.024 & 1.908 & 2,625 \\
\hline
\end{tabular}

Nota. * Quantidade de informação psicométrica entre -4 e +4 desvios.

\section{Análise de dados}

Por meio do software Factor 10.10.03 (Ferrando \& Lorenzo-Seva, 2017) averiguou-se a dimensionalidade da FCV-19S, tendo em conta o método Hull Comparative Fit Index (CFI; Lorenzo-Seva, Timmerman, \& Kiers, 2011), através de uma análise fatorial exploratória categórica DWLS (Diagonally Weighted Least Squares) robusta implementada na matriz de correlações policóricas. Ressalta-se que o método Hull configura-se como um dos melhores na estimação da dimensionalidade de um dado conjunto de itens (Lorenzo-Seva, Timmerman, \& Kiers, 2011). Além disso, verificou-se a consistência interna (precisão) pelo coeficiente alfa de Cronbach $(\alpha)$ com base nas correlações policóricas e pelo ômega $(\omega)$ de McDonald. Para tanto, considerou-se a escala de resposta do tipo Likert como categorias ordenadas (Holgado-Tello, Chacón-Moscoso, Barbero-García, \& Vila-Abad, 2010; Lara, 2014). Para que fossem avaliados os parâmetros dos itens da IPCS, por meio da Teoria de Resposta ao Item (TRI), foi empregado o software R com uso do pacote mirt (Chalmers, 2012). Ademais, levando em consideração a natureza politômica ordinal dos itens fez-se uso do Modelo de Resposta Graduada (GRM; Samejima, 1969).

\section{Resultados}

Inicialmente, buscou-se conhecer evidências de validade de construto a partir da estrutura fatorial da medida através de uma análise fatorial categórica robusta (500 reamostragens por meio de Bootstrap). Por oportuno, preliminarmente, observou-a adequação da matriz de correlações policóricas à AFE $[\mathrm{KMO}=.85$ (IC95\%=.837 $.841)$ e $\chi^{2}$ Bartlett $(210)=2.684,4$ e $\left.p<.001\right]$. Portanto, a AFE, usando DWLS, sem fixar número de fatores a extrair ou rotação, foi rodada, resultando em um único fator com valor próprio >1 (8.54) que explicou $40.66 \%$ da variância total, o que fora reforçado pelo método Hull (CFI=.95; Scree Test =33.40), além dos indicadores de unidimensionalidade (Ferrando \& Lorenzo-Seva, 

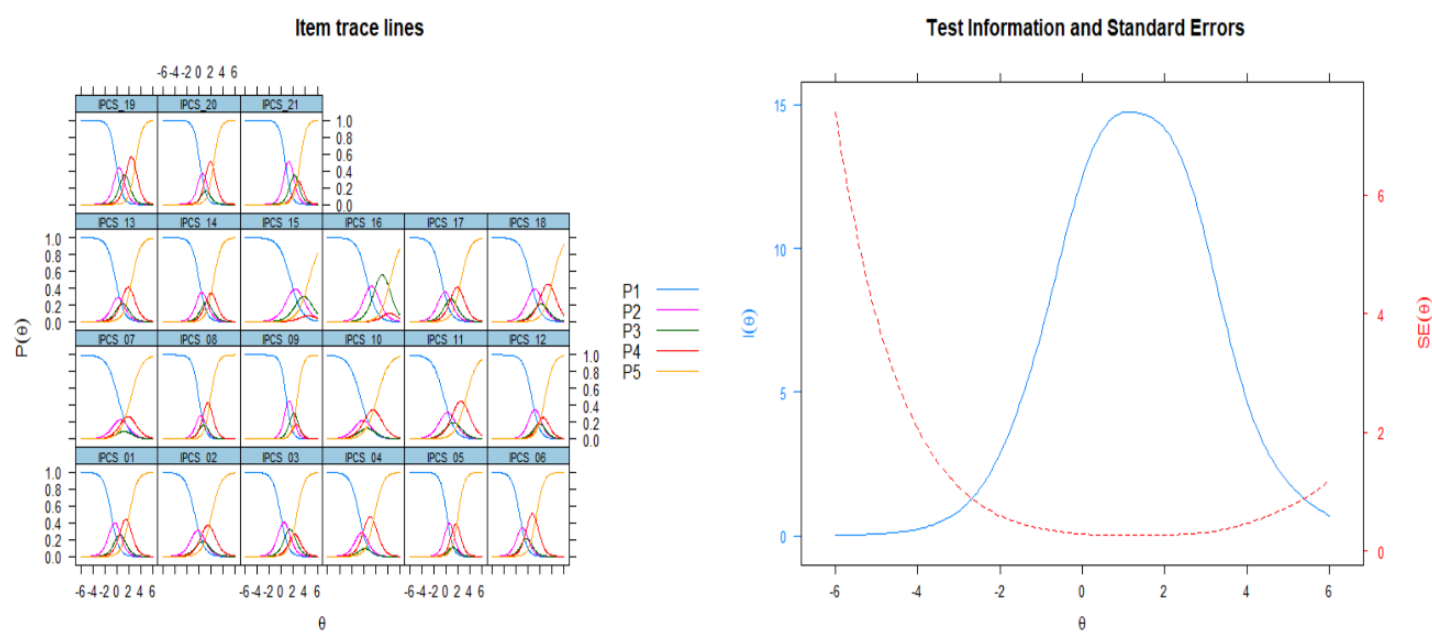

Figura 1. Curvas de informação dos itens e do teste

2018) UniCo (Unidimensional Congruence) $=.94$ $(\mathrm{IC} 95 \%=.923-.973)$ e MIREAL (Mean of Item Residual Absolute Loadings) $=.260$ (IC95\%=.193 .289). Adicionalmente, utilizou-se a TRI (GRM) para conhecer, com maiores detalhes, os parâmetros individuais dos itens e melhor avaliar a medida de perpetração do cyberstalking. No Quadro 1 encontram-se mais detalhes.

É possível observar no Quadro 1 que todos os 21 itens saturaram no fator perpetração do cyberstalking com cargas fatoriais superiores a .45 $(M=.63 ; D P=.07)$, variando de .47 (Item 15 . Se meu(minha) parceiro(a) me traísse, eu não hesitaria em usar as redes sociais para envergonhá-lo(a).) a .88 (Item 09. Já usei as configurações de localização em tempo real do celular/computador de meu (minha) parceiro(a) para ver onde ele(a) estava, sem ele(a) saber). Finalmente, a consistência interna avaliada pelo Alfa de Cronbach padronizado $(\alpha)$ e ômega de McDonald $(\omega=.90)$ foram iguais a .93 , índice considerado adequado, sendo superior ao recomendado na literatura ( $\geq .70$; Cortina, 1993; Taber, 2018).

No que tange à TRI, especificamente avaliouse a capacidade do item diferenciar pessoas no traço latente, limiares e quantidade de informação psicométrica. A capacidade média de discriminar pessoas $\left(M_{a}=1.49 ; D P_{a}=.33\right)$, variando de 1.02 (item 10) a 2.09 (Item 09), valores considerados entre moderados e altos (Baker, 2001; Baker \& Kim, 2017). Os limiares servem para identificar os níveis de theta $(\theta)$ necessários para que se endosse a categoria superior na escala de resposta, que geralmente indicam algo sobre a dificuldade do item, sendo valores baixos característicos de itens mais fáceis, enquanto níveis altos caracterizam itens considerados difíceis. No geral, os menores limiares $\left(b_{1-4}\right)$ ficaram por conta dos itens 06 e 04, tendo médias de $b_{1-4}$ de -1.22 e .38 respectivamente, portanto sendo os que requerem menores níveis de perpetração do cyberstalking para serem endossados completamente (ver Quadro 1 para mais detalhes). Por outro lado, os itens $15 \mathrm{e}$ 16 requerem níveis mais elevados de preparação para serem completamente endossados, apresentando os seguintes valores médios, respectivamente 3.40 e 2.81. Por fim, as curvas de informação dos itens e do teste foram avaliadas, podendo serem vistas graficamente na Figura 1.

Avaliando a quantidade de informação psicométrica $\mathrm{I}(\theta ;-4 /+4)$ dos itens individualmente (Quadro 1), percebe-se uma média de informação $\mathrm{I}(\theta)$ de $3,11(D P=1.02)$, com variabilidade de 1.79 (itens 07 e 10) a 4.57 (Item 19). A avaliação gráfica das curvas de informação dos itens e do teste permitem indicar que o teste cobre uma ampla faixa do traço latente (-1.41 a 4.33), além de ser possível classificar 10 itens como mais informativos (01, 03, 05, 06, 08, 09, 14, 19, 20 e 21) e, portanto, mais centrais para perpetração do cyberstalking. Neste sentido, sugere-se uma versão reduzida da IPCS, composta por 10 itens, também avaliada via EFA e TRI (ver Quadro 1).

Para a EFA para os 10 itens da IPCS, foram seguidas as mesmas especificações da realizada com a versão de 21 itens, e encontrou-se uma estrutura unidimensional (Eigenvalue $=5,08$; Hull $\left._{\mathrm{CFI}}=0,972\right)$, que apresentam cargas fatoriais acima de $.60(M=.68 ; D P=.05)$, sendo $(\alpha)$ e $(\omega)=.89$. Referente à discriminação dos itens, 


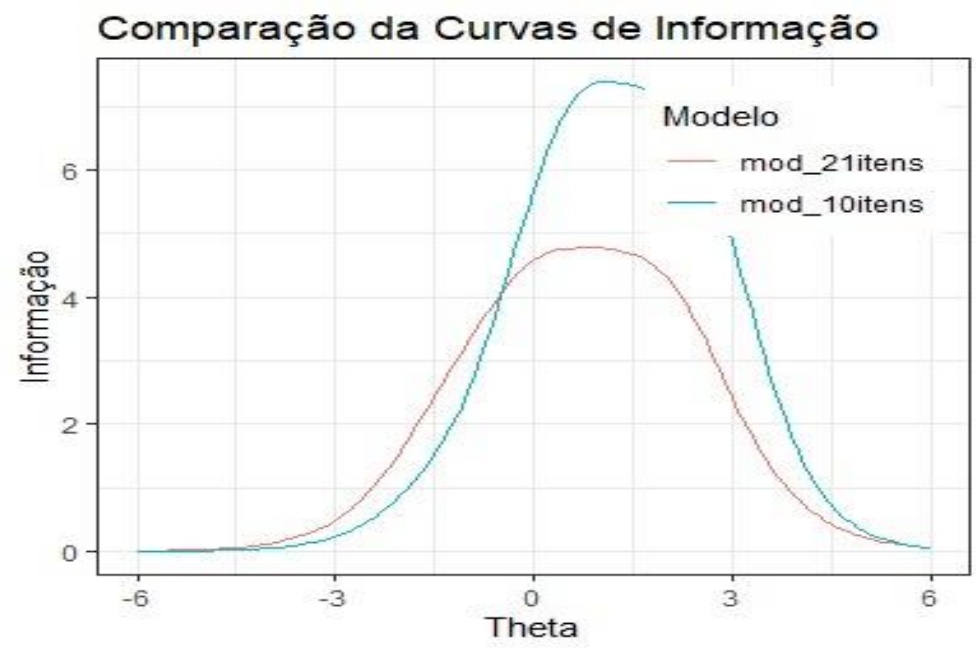

Figura 2. Comparação entre Curvas de Informação dos Testes com 21 e 10 itens

percebe-se valores de moderado (Item 06, $\mathrm{a}=1,43$ ) a muito alto (Item 19, a=3.33), com média 1.75 $(D P=.28)$. Já os limiares permitem indicar que os itens cobrem extensa amplitude do traço latente $\left(b_{1-4}\right.$ entre -1.42 e 3.33$)$, sendo o Item $02\left(b_{1-4}\right.$ médio=-.12) o que requer menor nível de traço latente para ser completamente endossado e o Item $21 \quad\left(b_{1-4}\right.$ médio=1.98) como o que requer maiores níveis. A Figura 2 expõe as curvas de informação do teste, em suas versões de 21 itens e 10 itens (reduzido) indicando que a versão mais curta pode ser uma alternativa plausível sem perda de informação psicométrica.

\section{Estudo 02- Comprovação da estrutura fatorial e validade convergente}

\section{Participantes}

A amostra foi composta por 225 pessoas da população geral de diferentes estados brasileiros ( $M_{\text {idade }}=26.18, D P=7.72$; amplitude 18 a 66 anos) de diferentes estados brasileiros, sendo em maioria dos estados da Paraíba (27.6\%), Piauí (20.4\%) e Ceará (10.7\%); com status de relacionamento namorando $(52 \%)$ e/ou casado/união estável $(2,1 \% \%)$, do sexo feminino $(58,7 \%)$, heterossexuais $(75,6 \%)$, com ensino superior incompleto (44\%) e renda familiar média de $5.463,32$ reais $(D P=900.000$ a $7.000,00)$. Estes foram angariados de forma não probabilística, por conveniência, utilizando a mesma estratégia de coleta de dados do estudo anterior.

\section{Instrumentos}

Os participantes responderam um livreto contendo os mesmos instrumentos descritos no Estudo 1, acrescendo-se:

Dark Triad Dirty Dozen (DTDD). Adaptada para o contexto brasileiro por Gouveia, Monteiro, Gouveia, Athayde e Cavalcanti (2016), constituída por 12 itens, distribuídos equitativamente em três fatores, tendo os itens como exemplo: "Costumo usar enganações ou mentiras para conseguir o que quero" (maquiavelismo), "Costumo ser cínico" (psicopatia) e "Costumo esperar favores especiais dos outros" (narcisismo). Estes itens são distribuídos equitativamente entre os fatores e são respondidos numa escala do tipo Likert variando de 1 (Discordo fortemente) e 5 (Concordo fortemente).

\section{Procedimento}

Adotou-se os mesmos procedimentos descritos no Estudo 1 e obedecidas as orientações previstas nas Resoluções 466/12 e 510/16 do Conselho Nacional de Saúde.

\section{Análise de dados}

Foram utilizados o SPSS-26 e o software R. Com o SPSS foram realizadas análises descritivas para descrever os participantes. Com o software R, por meio do pacote Lavaan (Rossel, 2012), executou-se uma análise fatorial confirmatória categórica (ordinal) com estimador Weighted Least Squares Mean and Variance-Adjusted (WLSMV; Muthén \& Muthén, 2014). Tal estimador é recomendado para dados ordinais e 

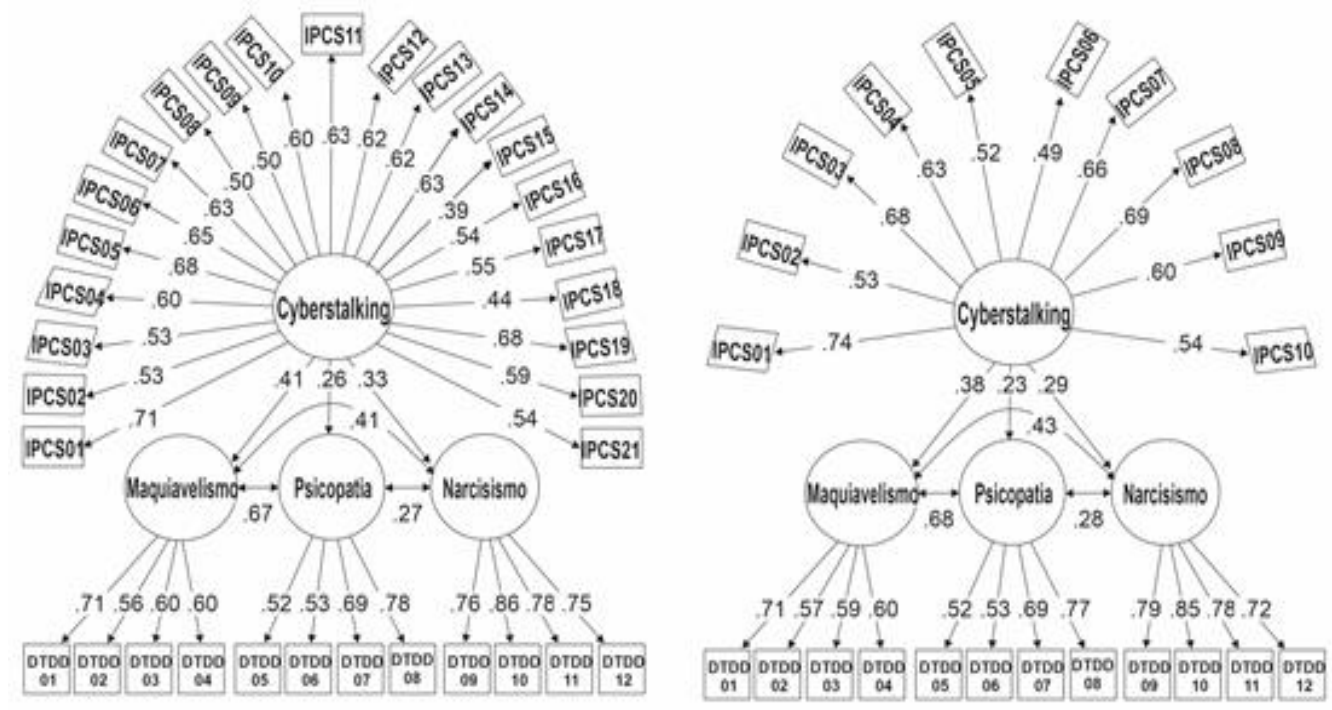

Figura 3. Validade convergente da IPCS em sua versão de 21 e 10 itens

que não seguem distribuição normal (Asún, RdzNavarro, \& Alvarado, 2015; Holgado-Tello et al., 2010), características idênticas às dos dados aqui utilizados.

Para tanto, avaliou-se o modelo proposto considerando os seguintes indicadores, geralmente recomendados (Byrne, 2010; Hair, Black, Babin, \& Anderson, 2019; Tabachnick \& Fidell, 2013): (1) Comparative Fit Index (CFI) - é um índice comparativo, valores a partir de .90 são referências de ajuste; (2) Tucker-Lewis Index (TLI) - medida de parcimônia entre os índices do modelo proposto e nulo, a qual varia de zero a um, com valores acima de .90 como aceitáveis; (3) Root-Mean-Square Error of Approximation (RMSEA) e seu intervalo de confiança de $90 \%$ (IC90\%), recomendando-se valores entre $.05 \mathrm{e}$ .08 , admitindo-se até .10 . Ademais, a precisão da medida foi calculada por meio do alfa de Cronbach e Ômega de McDonald, com o pacote semTools (semTools Contributors, 2016).

\section{Resultados}

\section{Análise Fatorial Confirmatória - AFC e validade convergente}

Para testar a estrutura unifatorial evidenciada no Estudo 1, tanto para a versão original (21 itens), quanto para a versão reduzida (10 itens), foram rodadas análises AFC's robustas, com estimador WLSMV. Os resultados indicaram bom ajuste para ambos os modelos testados, ou seja, o modelo original $[\mathrm{CFI}=.999 ; \quad \mathrm{TLI}=.996$; RMSEA $=.018$ (IC90\%=.035 - .026 e $p>.05$ ]; e o reduzido, representado por 10 itens [CFI=.999; TLI $=.989 ;$ RMSEA $=.021($ IC90\% $=.039-.050 \mathrm{e}$ $p>.05)]$. Seus indicadores de consistência interna são considerados altos (Kline, 2015), sugerindo boas evidências de fidedignidade (IPCS ${ }_{21 \text { itens, }} \alpha \mathrm{e}$ $\omega=.91$; IPCS ${ }_{10 \text { itens, }} \alpha=.86$ e $\omega=.85$ ).

Adicionalmente, para calcular a validade convergente recorreu-se à Modelagem por Equações Estruturais, com estimador WLSMV, coerente com a natureza das medidas utilizadas. Assim, foram dois modelos (um com a versão IPCS $_{21 \text { itens }}$ e outro com IPCS I0itens $_{\text {) }}$ com quatro variáveis latentes cada um: um representando a IPCS (composta pelos 21 ou 10 itens da IPCS, em modelos separados) e outros três representando maquiavelismo, psicopatia e narcisismo (cada um com 4 itens). Para o modelo com a versão da IPCS de 21 itens, observou-se que a variável latente da perpetração do cyberstalking apresentou peso de regressão como esperado, de modo positivo com os três outros construtos [maquiavelismo $\quad\left(\lambda_{\text {padronizado }}=.41 ; \quad p<.001\right)$, psicopatia $\left(\lambda_{\text {padronizado }}=.26 ; p<.05\right)$ e narcisismo $\left.\left(\lambda_{\text {padronizado }}=.33 ; p<.001\right)\right]$ e ajuste razoável [CFI=.91; TLI $=.90 ;$ RMSEA $=.034$ (IC90\% $=.024 /$ $.042 ; p=1.00)]$. Resultados na mesma direção foram encontrados para a versão de 10 itens, ou seja, a perpetração do cyberstalking foi explicada de forma positiva com maquiavelismo 
$\left(\lambda_{\text {padronizado }}=.38 ; \quad \quad p<.001\right), \quad$ psicopatia $\left(\lambda_{\text {padronizado }}=.23 ; \quad p<.05\right)$ e narcisismo ( $\left.\lambda_{\text {padronizado }}=.29 ; p<.001\right)$, com um modelo melhor ajustado quando comparado com o anterior $[\mathrm{CFI}=.93 ; \mathrm{TLI}=.93 ; \mathrm{RMSEA}=.036(\mathrm{IC} 90 \%=.001 /$ $.016 ; p=.80)]$. Para mais detalhes ver Figura 3.

\section{Discussão}

O cyberstalking por ser considerado um construto relativamente novo, ainda é pouco estudado, não havendo consenso se essa problemática representa uma forma modificada de stalking ou se é um fenômeno criminoso inteiramente novo e emergente, oriundo do contexto cibernético (Nobles, Reyns, Fox, \& Fisher, 2014). Neste contexto, sabe-se que ainda são escassas pesquisas empíricas sobre o cyberstalking, sendo a maioria direcionada às vítimas (Cavezza \& McEwan, 2014). Contrapondose a isto, considerando a perspectiva do agressor, pesquisas empíricas são relativamente recentes (March et al., 2020; Smoker \& March, 2017). Especificamente, considerando a perpetração no contexto dos relacionamentos íntimos e o papel de agressor (cyberstalkers), existe uma maior probabilidade deste grupo se envolver em condutas antissociais e delitivas (Goodboy \& Martin, 2015), a exemplo do cyberbullying e cybertrolling (Kircaburun et al., 2018).

Tais considerações reforçam a necessidade de se contar com instrumentos válidos e precisos que possibilitem avaliar e identificar adequadamente a prevalência e os possíveis antecedentes e consequentes desta conduta. Tais fatos encorajaram o desenvolvimento da presente pesquisa, que objetivou adaptar a Intimate Partner Cyberstalking Scale (IPCS) para o contexto brasileiro, reunindo evidências de validade e precisão. Dito isto, ressalta-se que apesar da IPCS ter sido utilizada em pesquisas prévias, a sua estrutura interna ainda não havia sido explorada, sendo necessário verificar a dimensionalidade do instrumento, como sugerido, respectivamente, por Smoker e March (2017) e March et al. (2020). Ressalta-se que a IPCS é uma das poucas ferramentas de avaliação da perpetração do cyberstalking.

Especificamente, no estudo 1 foram reunidas evidências acerca da estrutura unifatorial da medida, cujos itens apresentaram cargas fatoriais adequadas, considerando a carga fatorial $|\geq 0,40|$, ponto de corte que é considerado minimamente aceitável (Hair et al., 2009; Pasquali, 2012), além do coeficiente de consistência interna comprovar a sua precisão (Cortina, 1993; McDonald, 1999; Pasquali, 2016; Taber, 2018). Ademais, avaliando cada item individualmente, foi observado que os itens apresentaram discriminação adequada (Baker, 2001), sendo informativos e cobriram uma ampla gama da faixa do traço latente avaliado, ou seja, cyberstalking.

Considerando que a conduta agressiva do cyberstalking pela perspectiva do agressor ainda é pouco estudado, havendo apenas duas medidas psicométricas na literatura para avaliar tal conduta, sendo suas evidências psicométricas ainda pouco exploradas (Kircaburun, et al., 2018; March et al., 2020; Smoker \& March 2017), parece oportuno contar com empreendimentos como o da presente pesquisa, que possibilitem identificar medidas precisas e informativas para serem utilizadas em situações nas quais não se dispõe de muito tempo para avaliações ou triagens realizadas em contextos específicos, como o forense.

Portanto, foram selecionados os itens com maior informação psicométrica para compor a versão curta da Intimate Partner Cyberstalking Scale (IPCS). No caso, essa versão de 10 itens se mostrou tão informativa, válida e precisa quanto a versão de 21 itens, sendo uma alternativa útil para o rastreio de pessoas com potencial para apresentarem comportamentos de perpetração do cyberstalking.

No estudo 2, foi corroborada a estrutura unidimensional IPCS em suas duas versões (21 e 10 itens) que apresentaram indicadores de ajuste do modelo aos dados empíricos (e.g., CFI e TLI $\geq .95$ e RMSEA <.08; Tabachnick \& Fidell, 2013), com coeficientes adequados de consistência interna ( $\alpha$ e $\omega \geq .70$; McDonald, 1999; Taber, 2018). Além disso, foram verificadas evidências de validade com base em correlações com variáveis externas. Concretamente, verificouse que as duas versões de 21 e 10 itens da medida se associam na direção esperada e com pesos de regressão com magnitudes similares com maquiavelismo, psicopatia e narcisismo, desmonstrando evidências de validade convergente (Nunes \& Primi, 2010). 
Ademais, os modelos testados apresentaram indicadores de ajuste adequados (Marôco, 2014; Tabachnick \& Fidell, 2013). Assim, mesmo que a versão original composta por 21 itens tenha apresentado indicadores levemente mais favoráveis, a versão reduzida segue o critério da parcimônia (Pasquali, 2016) e avalia de forma similar o construto. Tais resultados são relevantes, pois demonstram evidências de validade referente à estrutura interna da IPCS. Suprem, assim, uma lacuna existente na literatura, além de corroborrar que a perpetração do cyberstalking pode ser uma conduta explicada pela personalidade sombria (Kircaburun et al., 2018; March et al., 2020).

Entretanto, apesar das evidências favoráveis, a presente pesquisa apresenta limitações, a exemplo da natureza transversal do presente estudo, que não permitiu avaliar a estabilidade temporal da IPCS. Além disso, o estudo atual se baseou em uma amostra reduzida $(<500)$, que não permite inferir generalizações para além da amostra considerada. Outra potencial limitação deste estudo se refere ao fato de terem sido consideradas medidas de autorrelato, fato que favorece o viés da desejabilidade social, inerente a construtos psicossociais (Gouveia, Guerra, Souza, Santos, \& Costa, 2009).

Devido estas considerações, sabe-se que estudos adicionais devem ser realizados a fim de averiguar evidências psicométricas adicionais da IPCS, tal como a validade externa da medida com diferentes construtos, já considerados em outros contextos, a exemplo do comportamento de trolagem e cyberbullying (Goodboy, \& Martin, 2015; Kircaburun et al., 2018), necessidade de controle (March et al., 2020) e violência doméstica (Sheldon, Rauschnabel, \& Honeycutt, 2019).

Ademais, seria interessante avaliar a estabilidade temporal da IPCS, por meio do testereteste ou resultados associados com outras medidas que avaliam as dimensão da tríade sombria (Pereira \& Paixão, 2019), além de considerar a personalidade sombria de forma mais abrangente, como o sadismo (Buckels \& Paulhus, 2013; O’Meara et al., 2011; Plouffe, Saklofske, \& Smith, 2017), que constitui a tétrade sombria, já considerada em estudos prévios que consideram as condutas desviantes no contexto online (Moor \& Anderson, 2019). Outrossim, seria igualmente interessante avaliar a sua invariância em diferentes grupos (por exemplo, considerando diferentes fases do desenvolvimento, tipos de relacionamento ou ter em conta amostras forenses).

Finalizando, acredita-se que o estudo contribuiu com o campo científico, pois reuniu evidências de validade, disponibilizando uma medida sobre perpetração do cyberstalking, na sua versão em português brasileiro, fornecendo uma medida mais curta e com qualidade comparável à versão original, podendo ser útil em pesquisas que considerem relacionar o cyberstalking com distintos construtos, que possibilitem compreender os possíveis antecedentes e consequências desta conduta, aumentando o escopo de pesquisas empíricas, incentivando estudos, principalmente em contexto brasileiro, que possivelmente possam traçar o perfil do agressor e incentivar a discussão de políticas públicas e leis que criminalizem o cyberstalking.

\section{Referências}

Ahlgrim, B., \& Terrance, C. (2018). Perceptions of cyberstalking: Impact of perpetrator gender and cyberstalker/Victim relationship. Journal of Interpersonal Violence. doi:10.1177/0886260518784590

Asún, R. A., Rdz-Navarro, K., \& Alvarado, J. M. (2015). Developing multidimensional likert scales using item factor analysis: The case of four-point items. Sociological Methods \& Research. doi:10.1177/0049124114566716

Baker, F. B. (2001). The basics of item response theory (2th ed.). Retrieved from http://eric.ed.gov/?id=ED458219

Baker, F. B., \& Kim, S. (2017). The basics of Item Response Theory using $R$. New York, NY: Springer.

Bocij, P., \& McFarlane, L. (2003). Cyberstalking: The technology of hate. The Police Journal, 76(3), 204-221. doi: 10.1350/pojo.76.3.204.19442

Borsa, J. C., Damásio, B. F., \& Bandeira, D. R. (2012) Adaptação e validação de instrumentos psicológicos entre culturas: Algumas considerações. Paidéia, 22(53), 423-432. doi:10.1590/S0103-863X2012000300014 
Buckels, E. E., Jones, D. N., \& Paulhus, D. L. (2013). Behavioral confirmation of everyday sadism. Psychological Science, 24(11), 22012209. doi:10.1177/0956797613490749

Byrne, B. M. (2010). Structural equation modeling with Amos: Basic concepts, applications, and programmimg (2th Ed.). New York, NY: Routledge.

Chalmers, R. P. (2012). mirt: A multidimensional item response theory package for the $\mathrm{R}$ environment. Journal of Statistical Software, 48(6), 1-29. doi:10.18637/jss.v048.i06

Cavezza, C., \& McEwan, T. E. (2014). Cyberstalking versus off-line stalking in a forensic sample. Psychology, Crime \& Law, 20(10), 955-970. doi:10.1080/1068316X.2014.893334

Cortina, J. M. (1993). What is coefficient alpha? An examination of theory and applications. Journal of Applied Psychology, 78(1), 98-104. doi:10.1037/0021-9010.78.1.98

DeTardo-Bora, K. A., and Bora, D. J. 2016. Cybercrimes: An overview of contemporary challenges and impending Threats. In J. Sammons (Ed.), Digital Forensics. Amsterdam: Elsevier, pp. 119-132.

Dreßing, H., Bailer, J., Anders, A., Wagner, H., \& Gallas, C. (2014). Cyberstalking in a large sample of social network users: Prevalence, characteristics, and impact upon victims. Cyberpsychology, Behavior, and Social Networking, 17(2), 61-67. doi:10.1089/cyber.2012.0231

Eterovic-Soric, B., Choo, K.-K. R., Ashman, H., \& Mubarak, S. (2017). Stalking the stalkers detecting and deterring stalking behaviours using technology: A review. Computers \& Security, $\quad 70, \quad 278-289$. doi:10.1016/j.cose.2017.06.008

Ferrando, P. J., \& Lorenzo-Seva, U. (2017). Program FACTOR at 10: Origins, development and future directions. Psicothema, 29, 236-240. doi:10.7334/psicothema2016.304

Ferrando, P. J., \& Lorenzo-Seva U. (2018). Assessing the quality and appropriateness of factor solutions and factor score estimates in exploratory item factor analysis. Educational and Psychological Measurement, 78, 762780. doi: $10.1177 / 0013164417719308$
Fox, K. A., Nobles, M. R., \& Fisher, B. S. (2011). Method behind the madness: An examination of stalking measurements. Aggression and Violent Behavior, 16(1), 74-84. doi:10.1016/j.avb.2010.12.004

Goodboy, A. K., \& Martin, M. M. (2015). The personality profile of a cyberbully: Examining the dark triad. Computers in Human Behavior, 49, 1-4. doi:10.1016/j.chb.2015.02.052

Gouveia, V. V., Guerra, V. M., Sousa, D. M. F., Santos, W. S., \& Costa, J. M. (2009). Escala de desejabilidade social de Marlowe-Crowne: Evidências de sua validade fatorial e consistência interna. Avaliação Psicológica, 8(1), 87-98.

Gouveia, V. V., Monteiro, R. P., Gouveia, R. S. V., Athayde, R. A. A., \& Cavalcanti, T. M. (2016). Avaliando o lado sombrio da personalidade: Evidências psicométricas do Dark Triad Dirty Dozen. Interamerican Journal of Psychology, 50, 420-432.

Hair, J. F., Black, W. C., Babin, B. J., \& Anderson, R. E. (2019). Multivariate Data Analysis. (8th ed.). Cengage Learning, U.K.

Holgado-Tello, F. P., Chacón-Moscoso, S., Barbero-García, I., \& Vila-Abad, E. (2010). Polychoric versus pearson correlations in exploratory and confirmatory factor analysis of ordinal variables. Quality \& Quantity, 44(1), 153-166. doi:10.1007/s11135-008-9190-y

Kline, P. (2015). A Handbook of Test Construction. New York, NY: Routledge.

Kircaburun, K., Jonason, P. K., \& Griffiths, M. D. (2018). The dark tetrad traits and problematic social media use: The mediating role of cyberbullying and cyberstalking. Personality and Individual Differences, 135, 264-269. doi:10.1016/j.paid.2018.07.034

Lara, S. A. D. (2014). ¿Matrices Policóricas/Tetracóricas o Matrices Pearson? Un estudio metodológico. Revista Argentina de Ciencias del Comportamiento, 6(1), 39-48.

Lyons, M. (2019). The dark triad of personality: Narcissism, machiavellianism, and psychopathy in everydayl. London: Academic Press. doi:10.1016/C2017-0-01262-4

Lorenzo-Seva, U., Timmerman, M. E., \& Kiers, H. A. L. (2011). The hull method for selecting the number of common factors. Multivariate 
Behavioral Research, 46(2), 340-364. doi:10.1080/00273171.2011.564527

March, E., Litten, V., Sullivan, D. H., \& Ward, L. (2020). Somebody that I (used to) know: Gender and dimensions of dark personality traits as predictors of intimate partner cyberstalking. Personality and Individual Differences, 163, 110084. doi:10.1016/j.paid.2020.110084

Marôco, J. (2014). Análise de equações estruturais: Fundamentos teoricos, software \& aplicações ( $2^{\mathrm{a}}$ ed.). Pero Pinheiro: Report Number.

McDonald, R. P. (1999). Test theory: A unified treatment. Mahwah, NJ: Erlbaum.

Ménard, K. S., \& Pincus, A. L. (2012). Predicting overt and cyber stalking perpetration by male and female college students. Journal of Interpersonal Violence, 27(11), 21832207. doi:10.1177/0886260511432144

Moor, L., \& Anderson, J. R. (2019). A systematic literature review of the relationship between dark personality traits and antisocial online behaviours. Personality and Individual Differences, 144, 40-55. doi:10.1016/j.paid.2019.02.027

Muthén, L. K., \& Muthén, B. O. (2014). Mplus user's guide. (7th Ed.). Los Angeles: Muthén \& Muthén.

Nobles, M. R., Reyns, B. W., Fox, K. A., \& Fisher, B. S. (2012). Protection against pursuit: A conceptual and empirical comparison of cyberstalking and stalking victimization among a national sample. Justice Quarterly, 31(6), 9861014. doi:10.1080/07418825.2012.723030

Nunes, C. H. S. S., Primi, R., \& Conselho Federal de Psicologia. (2010). Aspectos técnicos e conceituais da ficha de avaliação dos testes psicológicos. Avaliação psicológica: Diretrizes na regulamentação da profissão, 101-128.

Retrieved

from https://satepsi.cfp.org.br/docs/Diretrizes.pdf

O'Meara, A., Davies, J., \& Hammond, S. R. (2011). The psychometric properties and utility of the Short Sadistic Impulse Scale (SSIS). Psychological Assessment, 23(2), 523531. doi:10.1037/a0022400
Pasquali, L. (2012). TRI - Teoria de resposta ao item: teoria, procedimentos e aplicações. Curitiba, PR: Apriss editora.

Pasquali, L. (2016). TEP - Técnicas de exame psicológico: Os fundamentos. ( $2^{\mathrm{a}}$ ed.). São Paulo, SP: Vetor editora.

Pereira, C., \& Paixão, R. (2019). Estrutura Fatorial da Versão Portuguesa da Escala de Narcisismo Hipersensível. Revista Iberoamericana de Diagnóstico y Evaluación - e Avaliação Psicológica, 53(4), 19-31.

Pires, S., Sani, A., \& Soeiro, C. (2018). Stalking e ciberstalking em estudantes universitários: Uma revisão sistemática. Revista Portuguesa de Investigação Comportamental e Social 4(2), 60-75. doi: 10.31211/rpics.2018.4.2.75

Plouffe, R. A., Saklofske, D. H., \& Smith, M. M. (2017). The assessment of sadistic personality: Preliminary psychometric evidence for a new measure. Personality and Individual Differences, 104, 166171. doi:10.1016/j.paid.2016.07.043

Reyns, B. W. (2018). Online pursuit in the twilight zone: Cyberstalking perpetration by College Students. Victims \& Offenders, 116. doi:10.1080/15564886.2018.1557092

Rossel, P. (2012). Early detection, warnings, weak signals and seeds of change: A turbulent domain of futures studies. Futures, 44(3), 229239.

https://doi.org/10.1016/j.futures.2011.10.005

Ruiz-García, A., Jiménez, O, Fenollar-Cortés, J., Cano-Marín, J., Ojeda-Mora, M. A., Selva, A., \& Valero-Aguayo, L. (2019). Adaptación y validación al español del Cuestionario de Ansiedad por Separación en el Adulto (ASA27). Revista Iberoamericana de Diagnóstico y Evaluación -- e Avaliação Psicológica, 53(4), 153-167.

Samejima, F. (1969). Estimation of latent ability using a response pattern of graded scores. Psychometrika Monograph Supplement, 34(4, Pt. 2), 100.

Sheldon, P., Rauschnabel, P. A., \& Honeycutt, J. M. (2019). Cyberstalking and bullying. The dark side of social media, 4358. doi:10.1016/b978-0-12-815917-0.00003-4

Sheridan L. P., Blaauw, E., \& Davies G. M. (2003). Stalking: Knowns and unknowns. Trauma Violence Abuse, 4(2), 148-62. 
doi:10.1177/1524838002250766

Smith-Darden, J. P., Kernsmith, P. D., Victor, B. G., \& Lathrop, R. A. (2017). Electronic displays of aggression in teen dating relationships: Does the social ecology matter? Computers in Human Behavior, 67, 33-40. doi:10.1016/j. chb.2016.10.015

Smoker, M., \& March, E. (2017). Predicting perpetration of intimate partner cyberstalking: Gender and the dark tetrad. Computers in Human Behavior, doi:10.1016/j.chb.2017.03.012

Spitzberg, B. H., \& Cupach, W. R. (2003). What mad pursuit? Obsessive relational intrusion and stalking related phenomena. Aggression and Violent Behavior, 8(4), 345-375. doi:10.1016/S1359-1789(02)00068-X

Spitzberg, B., \& Cupach, W. (2007). The state of the art of stalking: Taking stock of the emerging literature. Aggression and Violent Behavior, 12(1), 64-86. doi:10.1016/j.avb.2006.05.001

Strawhun, J., Adams, N., \& Huss, M. T. (2013). The assessment of cyberstalking: An expanded examination including social networking, attachment, jealousy, and anger in relation to violence and abuse. Violence and Victims, 28(4), 715-730. doi:10.1891/0886-6708.11-00145

Tabachnick, B. G., \& Fidell, L. S. (2013). Using multivariate statistics (6th ed..). Boston, MA: Pearson.

Taber, K. S. (2018). The use of cronbach's alpha when developing and reporting Research Instruments in science education. Research in Science Education, 48, 1273-1296 https://doi.org/10.1007/s11165-016-9602-2

Tani, F., Greenman, P. S., Schneider, B. H., \& Fregoso, M. (2003). Bullying and the big five: A study of childhood personality and participant roles in bullying incidents. School Psychology International, 24(2), 131-146. doi: 10.1177/0143034303024002001

Tokunaga, R. S. (2016). Interpersonal surveillance over social network sites: Applying a theory of negative relational maintenance and the investment model. Journal of Social and Personal Relationships, 33, 171-190. doi:10.1177/0265407514568749
Tokunaga, R. S., \& Aune, K. S. (2015). Cyberdefense: A taxonomy of tactics for managing cyberstalking. Journal of Interpersonal Violence, 32(10), 1451-1475. doi:10.1177/0886260515589564

Wegmann, E., Oberst, U., Stodt B., \& Brand, M. (2017). Online-specific fear of missing out and Internet-use expectancies contribute to symptoms of Internet-communication disorder. Addictive Behaviors Reports, 14(5), 33-42. doi:10.1016/j.abrep.2017.04.001 